\title{
NEAR-ULTRAVIOLET SOURCES IN THE HUBBLE ULTRA DEEP FIELD: THE CATALOG
}

\author{
Elysse N. Voyer ${ }^{1,2,5}$, Duilia F. de Mello ${ }^{1,2,6}$, Brian Siana $^{3}$, Jonathan P. Gardner ${ }^{2}$, Cori Quirk ${ }^{1}$, And Harry I. TePlitz ${ }^{4}$ \\ ${ }^{1}$ The Catholic University of America, Washington, DC 20064, USA \\ ${ }^{2}$ Observational Cosmology Laboratory, Goddard Space Flight Center, Code 665, Greenbelt, MD 20771, USA \\ ${ }^{3}$ California Institute of Technology, MS 105-24, Pasadena, CA 91125, USA \\ ${ }^{4}$ Spitzer Science Center, California Institute of Technology, MS 220-6, Pasadena, CA 91125, USA \\ Received 2009 February 20; accepted 2009 June 1; published 2009 July 7
}

\begin{abstract}
The catalog from the first high-resolution $U$-band image of the Hubble Ultra Deep Field, taken with Hubble's Wide-Field Planetary Camera 2 through the F300W filter, is presented. We detect $96 U$-band objects and compare and combine this catalog with a Great Observatories Origins Deep Survey B-selected catalog that provides $B$, $V, i$, and $z$ photometry, spectral types, and photometric redshifts. We have also obtained far-ultraviolet (FUV, 1614 Å) data with Hubble's Advanced Camera for Surveys Solar Blind Channel (ACS/SBC) and with GALEX. We detected 31 sources with ACS/SBC, 28 with GALEX/FUV, and 45 with GALEX/NUV. The methods of observations, image processing, object identification, catalog preparation, and catalog matching are presented.
\end{abstract}

Key words: galaxies: evolution - galaxies: formation - galaxies: starburst

Online-only material: color figures

\section{INTRODUCTION}

The Hubble Ultra Deep Field (UDF) campaign (Beckwith et al. 2006) has produced the deepest optical images of our universe to date. The UDF was observed by the Hubble Space Telescope (HST) in 412 orbits that were centered in a region of the Chandra Deep Field-South (CDF-S), which was also the target of the GOODS (Giavalisco et al. 2004) known as the GOODS-South or GOODS-S. The UDF used the same Advanced Camera for Surveys (ACS) filters as GOODS, F435W $\left(B_{435}\right), \mathrm{F} 606 \mathrm{~W}\left(V_{606}\right), \mathrm{F} 775 \mathrm{~W}\left(i_{775}\right)$, and F850LP $\left(z_{850}\right)$, but covered only one field of the 15 GOODS-S fields. The UDF reached approximately uniform limiting magnitudes $m_{\mathrm{AB}} \sim 29$ for point sources, at least 2 mag deeper than GOODS. Both campaigns, the UDF and the GOODS, did not include deep imaging in the $U$ bandpass. Taking $U$-band photometry is a time-consuming task because longer integrations are required to achieve comparable depth to optical images. Only three HST $U$-band deep fields have been taken so far, the original Hubble Deep Field, the Hubble Deep Field South, and the deepest $U$ band, with $H S T$ which were part of the parallel campaign of the UDF and lies on the edge of the GOODS-S (Williams et al. 1996; Casertano et al. 2000; de Mello et al. 2006b). GOODS has only partial $U$-band coverage with $H S T$ obtained during parallel observations (de Mello et al. 2006a). Deep $U$-band ground-based images of the GOODS-S field, such as those taken with the CTIO $4 \mathrm{~m}$ and the ESO $2.2 \mathrm{~m}$ available in the GOODS Web site ${ }^{7}$ are included in the multiwavelength coverage of GOODS. Although ground-based observations can more efficiently cover the larger fields of view than Hubble's cameras, they do not possess the same angular resolution as space-based observations. Low-resolution ground-based images will blend together nearby detections leading to inaccurate photometric redshifts and morphological analysis. The $U$ band is a critical wavelength in studies at intermediate redshifts $(z<2)$ since the

\footnotetext{
5 NASA Graduate Student Research Program Fellow.

6 Visiting Scientist in the Department of Physics and Astronomy, Johns Hopkins University, Baltimore, MD 21218, USA.

7 http://www.stsci.edu/science/goods/SupportObs/cdfs_mosaic/
}

rest-frame UV light is redshifted into the $U$ bandpass. It is in the UV that short-lived, massive, $\mathrm{O}$ and $\mathrm{B}$ stars radiate most of their energy and therefore the $U$ band is necessary to probe the unobscured star formation activity in galaxies at $z<2$.

After a redshift of $z \sim 1.5-2$, the star formation rate (SFR) of the universe began to steadily decline, decreasing by more than an order of magnitude (e.g., Hopkins \& Beacom 2006; Wadadekar et al. 2006). However, it is still an open question as to what population of objects contributes to the SFR density during the decline, and whether downsizing (the shift in star formation being dominated from large to small mass galaxies as the universe aged) plays an important role in this era (Cowie et al. 1996; Savaglio et al. 2005; Mouri \& Taniguchi 2006; Neistein et al. 2006). Therefore, studies that use $U$-band observations to better understand the nature of star-forming galaxies at intermediate $-z$ can greatly contribute to connecting the early universe and the local universe.

In this paper, we present the first $H S T$-targeted $U$-band image of the UDF. We present the $U$-band object catalog, and describe the methods used for observations, image processing, object identification, and catalog preparation.

\section{OBSERVATIONS}

The $U$-band observations were obtained with the HST WideField Planetary Camera 2 (WFPC2) in the Cycle 13 HST Treasury proposal (Teplitz, Program 10403). The UDF was imaged in the near-UV (NUV) through the WFPC2/F300W filter $\left(\lambda_{\max }=2987 \AA\right.$ and $\left.\Delta \lambda=740 \AA\right)$ in $12 H S T$ orbits divided into 4 roll angles to compensate for the shape of the WFPC2 chevron and achieve uniform depth. On-chip binning $(2 \times 2)$ was applied during WFPC 2 observations to reduce the effects of read-out noise, i.e., each wide field became $400 \times 400$ pixels. A total of 24 WFPC 2 images were taken with individual exposure times of $1200 \mathrm{~s}$.

FUV imaging was also obtained in this proposal with HST/ ACS Solar Blind Channel (SBC) camera at which time WFPC2 parallel observations were also made. The UDF ACS/SBC images were taken in $50 \mathrm{HST}$ orbits using the long-pass quartz filter (F150LP; $\lambda_{\text {eff }}=1614 \AA$ and FWHM $=177 \AA$ ). The FUV 


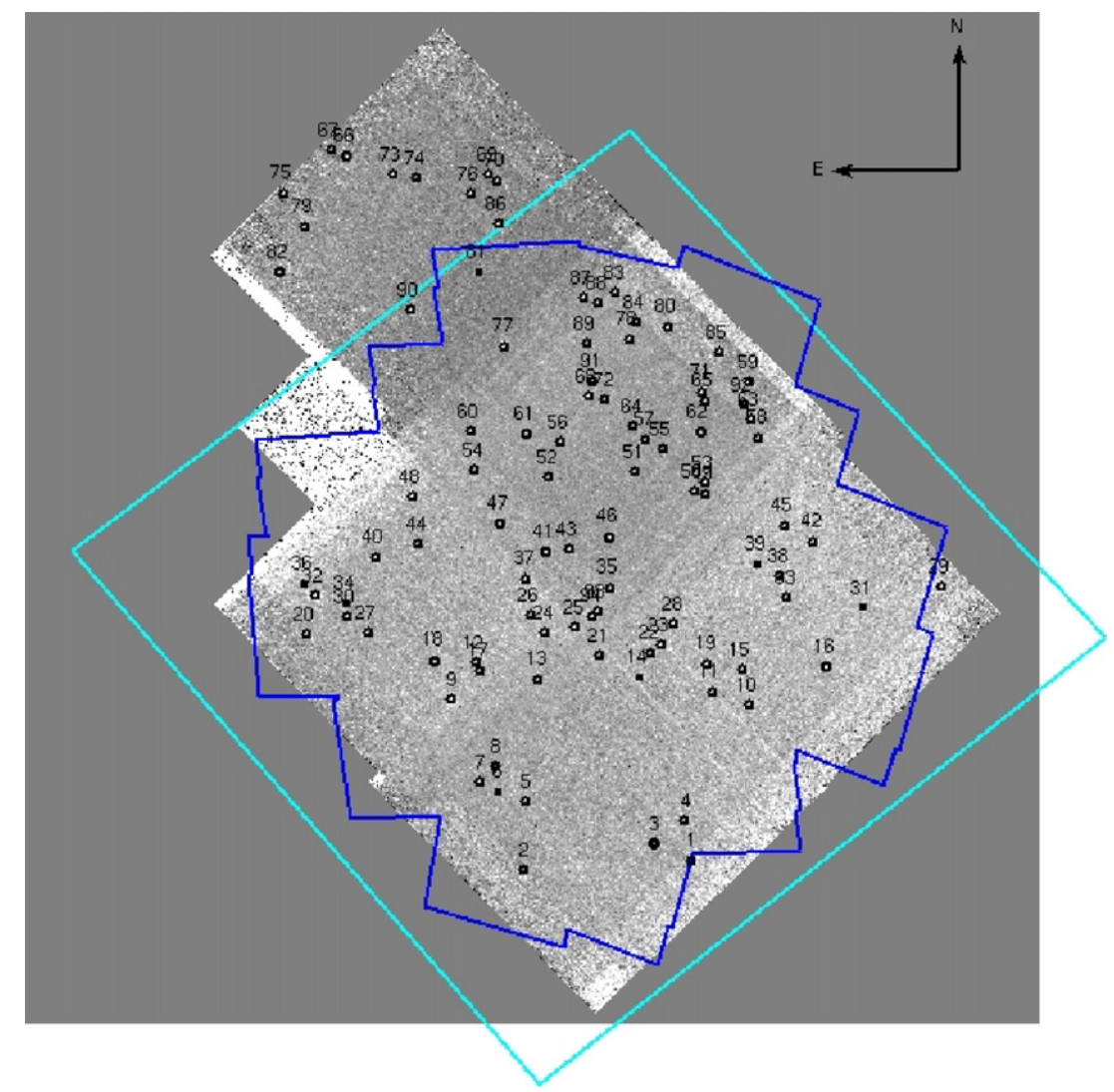

Figure 1. WFPC2 drizzled $U$-band image overlayed with UDF (square) and ACS/SBC (jagged) footprints. Note the large number of detections in the second rectangular region from the top of the image due to a higher net exposure time for this region.

(A color version of this figure is available in the online journal.)

was imaged in 25 pointings. Each pointing had a four point dither pattern with two $650 \mathrm{~s}$ exposures at each dither position. The total exposure time per pointing was $\sim 5200 \mathrm{~s}$ (Siana et al. 2007).

\section{IMAGE PROCESSING}

The WFPC2 images were retrieved from the $H S T$ archive for further processing. We combined the 24 WFPC 2 images with the MultiDrizzle code in the PyDrizzle package (Koekemoer et al. 2002). These images were taken using a dithering technique that reduces effects of pixel-to-pixel errors and allows one to better remove hot pixels, bad columns, and charge traps from the image. Dithering also allows the recovery of information lost to undersampling by pixels that are not small compared to the point-spread function (PSF). MultiDrizzle simplifies and automates the detection of cosmic rays of these dithered observations. We used calibrated flat fields from the HST pipeline and ran the MultiDrizzle script through the following steps. First, a static mask was created to identify bad pixels, then each image was sky-subtracted; shifts were determined from header coordinates for each image and were applied in drizzling each image separately onto registered output images. Next, a median image was created from these separate drizzled images and was blotted back to each original input image. Finally, the blotted images were used to compute cosmic-ray masks, and the final drizzle combination was performed using these masks.

Prior to running the MultiDrizzle, the Planetary Camera (PC) data were removed from all images because its inclusion greatly increased the noise level of the output drizzled image. This was achieved by replacing the PC in each of the 24 images with hot pixels, forcing the MultiDrizzle to automatically include the PC in the pixel mask for each individual image. We set the userinputs to MultiDrizzle so the script would output separate science and weight images for these data that we have made available online at http://goods.gsfc.nasa.gov/release/UDF_F300W.

The drizzled WFPC2 image shown in Figure 1 has a total exposure time of $28,800 \mathrm{~s}$ and a pixel scale of $0.1 \mathrm{arcsec}$. Due to the nature in which the 24 WFPC2 pointings were positioned, the final drizzled image does not have a uniform depth. The majority of pointings overlap in the mid-upper region of the combined image. Consequently, the majority of UV sources are detected in this area of the UDF. Also, the uppermost section of the $U$-band image lies just outside the UDF footprint, and sources from this area are marked as such in the catalog. The reduction procedure used for the UDF ACS/SBC images is outlined in Teplitz et al. (2006).

\section{OBJECT IDENTIFICATION AND CATALOG PREPARATION}

The catalog of $U$-band sources was produced using SourceExtractor version 2.5 (hereafter SE; Bertin \& Arnouts 1996). Initially, we created both a low- $\sigma$ ( $1.5 \sigma$ above background noise) and a high- $\sigma$ ( $3 \sigma$ above background noise) catalog. The high- $\sigma$ catalog contains all visually confirmed sources in the image, while the low- $\sigma$ catalog may contain spurious detection. The difference between the SE parameters specified for these two catalogs was the detection threshold relative to the background rms. For both catalogs, the minimum area of adjoining pixels for a detection was 15 pixels, and the minimum deblending parameter was set at $10 \%$, except for a few cases where we set 


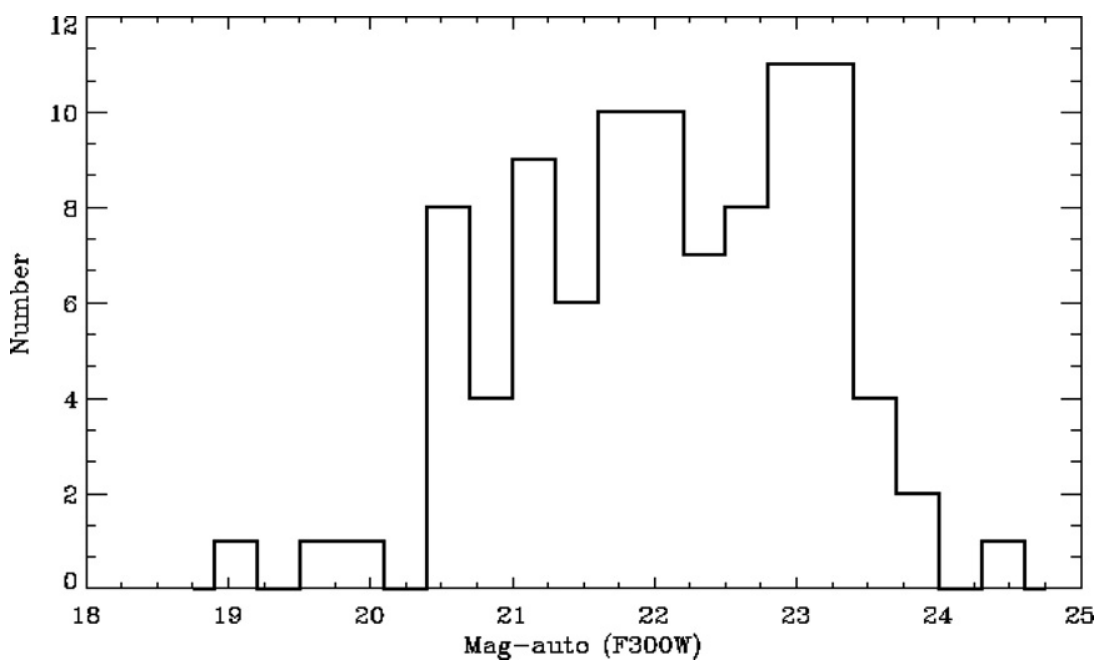

Figure 2. Magnitude distribution of the $U$-band catalog, MAG_AUTO from SE.

the deblending value in order to avoid multiple detections in one single object. This is particularly critical for source detection in $U$-band images since star-forming regions can appear as multiple clumps in one object. SE might detect those clumps as individual objects making false identifications.

The GAIN, MAG_ZEROPOINT, and SATUR_LEVEL parameters which are specific to the WFPC2 camera were set to $7 e^{-} / \mathrm{ADU}, 20.77 \mathrm{mag}$, and $2 \mathrm{ADU} \mathrm{s}^{-1}$, respectively. The weight map produced by the drizzling process was used by setting the weight map type to MAP_WEIGHT. Photometric measurements of each source were calculated using SE's automatic aperture magnitudes (MAG_AUTO). MAG_AUTO uses a Kron (1980) flexible elliptical aperture to measure the total magnitude of each source. Instead of using the classical aperture photometry with a fixed aperture radius, MAG_AUTO has $_{-}$ the advantage of limiting the background noise while detecting light from faint sources more effectively. The size of the background mesh which is subtracted from the photometry of each source (BACK_SIZE) was set to 64 pixels, and its rms value is used to calculate photometric errors. We cleaned the high- $\sigma$ and low- $\sigma$ catalogs removing sources with photometric errors $\geqslant 1.0$.

The resulting $U$-band catalog includes all detections from the high- $\sigma$ catalog, and the remaining objects visually confirmed from the low- $\sigma$ catalog. The $U$-band $1.5 \sigma$ limiting magnitude measured within a $1^{\prime \prime}$ diameter aperture is 23.5 mag (AB; Figure 2).

\subsection{Visual Identification of U-Band Sources}

We have visually checked each SE $U$-band detection in order to decide (1) if a single source detection is actually multiple sources, (2) if multiple source detections are single sources, (3) if a detection is too noisy, or (4) if there are any faint UV sources which are not detected. When such cases occur, SE parameters can be adjusted to maximize $U$-band source detections in the UDF image, and nondetections can be omitted from the catalog.

We have also visually identified the $U$-band sources within a Hubble ACS $/ B$-band image of the GOODS-S field that overlaps the UDF. The $B$-band sources had been cataloged by the GOODS team using SE and have matched aperture photometry in multiple ACS bands $(V, i, z)$ (Dahlen et al. 2007). The $B$-band catalog also lists spectral types (see Section 5 for definition) and photometric redshifts with a typical GOODS accuracy of $\Delta_{z}=$
0.8 (where $\Delta_{z} \equiv\left\langle\left|z_{\text {phot }}-z_{\text {spec }}\right| /\left(1+z_{\text {spec }}\right)\right\rangle$; Dahlen et al. 2007) for each source. If a $U$-band detection could not be visually identified as one of the objects in the $B$-band catalog, it was removed from the $U$-band catalog. We did this because the $B$-band data are much deeper (limiting $10 \sigma$ sensitivity is 27.8 ; Giavalisco et al. 2004) than the $U$ band, and we would not expect to detect a source in the $U$ band without also seeing it in the $B$ band. During this cleaning, the majority of spurious $U$-band detections located in the borders of the WFPC2 image were removed. We also discovered five $B$-band objects that corresponded to multiple detections in the $U$ band. This was a result of setting SE's deblending parameters to a low value in an effort to detect as many $U$-band sources as possible. This parameter was adjusted in an additional SE run to obtain single detections of these sources. The final $U$-band catalog contains 96 objects.

\subsection{Catalog Matching}

We matched the final $U$-band catalog to the far-UV catalog of the UDF created from the ACS/SBC observations described in Section 2 (Siana et al. 2007). Each ACS/SBC source was matched to the nearest $U$-band object within a 2 ".5 radius. The typical difference between WFPC2/F300W coordinates (R.A. and decl.) and ACS/SBC coordinates of the same object is 0 .'1. Thirteen $U$-band sources do not have FUV detections because they are outside the ACS/SBC footprint. Any ACS/ SBC sources with signal-to-noise ratio $(\mathrm{S} / \mathrm{N})<3 \sigma$ were not considered. In total, 31 of the $96 U$-band objects have resolved matching ACS/SBC detections.

The UDF has also been observed with the Galaxy Evolution Explorer (GALEX) mission in the far- and near-ultraviolet (FUV: $\lambda_{\text {eff }}=1528 \AA, \Delta \lambda_{\text {FUV }}=269 \AA$; NUV: $\lambda_{\text {eff }}=2271 \AA, \Delta \lambda_{\mathrm{NUV}}$ $=616 \AA$; GALEX field of view is 1.28 and 1.24 in FUV and NUV, respectively, and pixel scale is $1^{\prime \prime} .5$ pixel $\left.^{-1}\right)$ and is publicly available in the GALEX Release 4 (GR4) at the Multimission Archive at STScI (MAST). These data are from two different surveys, the All Sky Survey (AIS; 4".3 FWHM) and the Deep Sky Survey (DIS; 5".3 FWHM). The $5 \sigma$ limiting magnitudes of the AIS data are 20.8 in the NUV and 19.9 in the FUV, and for the DIS data 24.4 in the NUV and 24.8 in the FUV (Morrissey et al. 2007).

We searched for all $U$-band objects in the GR4 and found 30 detected by GALEX/FUV and 59 in the NUV. Since GALEX 
Table 1

UDF $U$-Band Sources

\begin{tabular}{|c|c|c|c|c|c|c|c|c|c|c|c|c|}
\hline $\begin{array}{l}\text { R.A. } \\
\text { (deg) }\end{array}$ & $\begin{array}{l}\text { Decl. } \\
\text { (deg) }\end{array}$ & $U_{\mathrm{mag}}$ & $\mathrm{FUV}_{\mathrm{mag}}$ & $B_{\mathrm{mag}}$ & $V_{\mathrm{mag}}$ & $i_{\mathrm{mag}}$ & $z_{\mathrm{mag}}$ & $\begin{array}{c}\text { GALEX } \\
\text { NUV }_{\text {mag }}\end{array}$ & $\begin{array}{l}\text { GALEX } \\
\text { FUV }_{\text {mag }}\end{array}$ & $z_{\text {phot }}$ & $z_{\text {spec }}$ & $\overline{\mathrm{ST}}$ \\
\hline 53.1306038 & -27.7902641 & $20.50 \pm 0.05$ & $\ldots$ & $22.46 \pm 0.02$ & $21.80 \pm 0.01$ & $21.16 \pm 0.01$ & $21.01 \pm 0.01$ & $23.52 \pm 0.07$ & $25.69 \pm 0.25$ & 0.66 & $\ldots$ & 3.67 \\
\hline 53.1376457 & -27.7919559 & $21.70 \pm 0.07$ & $\ldots$ & $23.54 \pm 0.04$ & $23.13 \pm 0.02$ & $22.44 \pm 0.03$ & $22.04 \pm 0.02$ & $\ldots$ & $\ldots$ & 0.85 & $\ldots$ & 3.67 \\
\hline 53.1409721 & -27.7966537 & $22.12 \pm 0.05$ & $24.97 \pm 0.06$ & $23.54 \pm 0.04$ & $22.47 \pm 0.01$ & $21.95 \pm 0.02$ & $21.69 \pm 0.02$ & $24.63 \pm 0.24$ & $\cdots$ & 0.41 & 0.3500 & 3.00 \\
\hline 53.1421356 & -27.7866974 & $21.02 \pm 0.03$ & $23.55 \pm 0.03$ & $22.11 \pm 0.01$ & $21.15 \pm 0.01$ & $20.73 \pm 0.01$ & $20.57 \pm 0.01$ & $23.18 \pm 0.09$ & $23.90 \pm 0.16$ & 0.35 & 0.2262 & 3.33 \\
\hline 53.1445389 & -27.7911339 & $21.58 \pm 0.06$ & $\ldots$ & $24.22 \pm 0.07$ & $24.16 \pm 0.05$ & $24.04 \pm 0.09$ & $23.84 \pm 0.09$ & $\ldots$ & $\ldots$ & 1.80 & $\ldots$ & 6.00 \\
\hline 53.1447334 & -27.7854404 & $20.97 \pm 0.03$ & $23.82 \pm 0.04$ & $23.20 \pm 0.02$ & $22.58 \pm 0.01$ & $22.47 \pm 0.02$ & $22.51 \pm 0.02$ & $23.44 \pm 0.07$ & $23.78 \pm 0.08$ & 0.07 & $\ldots$ & 6.00 \\
\hline 53.1450996 & -27.7894268 & $23.19 \pm 0.11$ & $\ldots$ & $24.15 \pm 0.06$ & $23.96 \pm 0.04$ & $23.58 \pm 0.06$ & $23.07 \pm 0.05$ & & $\ldots$ & 0.84 & $\ldots$ & 5.33 \\
\hline $53.1470947^{c}$ & -27.7784290 & $22.46 \pm 0.08$ & & $24.43 \pm 0.06$ & $24.28 \pm 0.04$ & $23.93 \pm 0.06$ & $23.73 \pm 0.06$ & $24.91 \pm 0.25$ & $\ldots$ & 1.12 & $\ldots$ & 6.00 \\
\hline 53.1472054 & -27.7884884 & $22.71 \pm 0.08$ & $26.29 \pm 0.16$ & $24.52 \pm 0.06$ & $23.99 \pm 0.03$ & $23.35 \pm 0.04$ & $23.19 \pm 0.04$ & $\ldots$ & $\cdots$ & 0.73 & $\ldots$ & 4.00 \\
\hline 53.1477814 & -27.7769451 & $21.87 \pm 0.07$ & $\ldots$ & $24.74 \pm 0.06$ & $24.66 \pm 0.05$ & $23.90 \pm 0.05$ & $23.43 \pm 0.04$ & $\ldots$ & $\ldots$ & 1.07 & 1.0860 & 3.67 \\
\hline $53.1478577^{\mathrm{c}}$ & -27.7740345 & $20.58 \pm 0.03$ & $\ldots$ & $22.59 \pm 0.02$ & $22.33 \pm 0.01$ & $21.76 \pm 0.02$ & $21.30 \pm 0.01$ & $23.03 \pm 0.07$ & $\ldots$ & 1.02 & $\ldots$ & 4.00 \\
\hline 53.1479225 & -27.7996845 & $23.00 \pm 0.10$ & $26.13 \pm 0.09$ & $25.06 \pm 0.15$ & $24.35 \pm 0.07$ & $23.75 \pm 0.08$ & $23.58 \pm 0.08$ & $\ldots$ & $\ldots$ & 0.67 & $\ldots$ & 4.00 \\
\hline 53.1484451 & -27.7757874 & $22.80 \pm 0.07$ & $25.56 \pm 0.09$ & $24.14 \pm 0.04$ & $23.24 \pm 0.02$ & $22.68 \pm 0.02$ & $22.45 \pm 0.02$ & $22.75 \pm 0.10$ & $\ldots$ & 0.48 & 3.6664 & 3.33 \\
\hline 53.1485405 & -27.7968845 & $22.48 \pm 0.08$ & $\ldots$ & $23.56 \pm 0.03$ & $23.52 \pm 0.03$ & $23.49 \pm 0.05$ & $23.43 \pm 0.05$ & $\ldots$ & $\ldots$ & 1.84 & $\ldots$ & 6.00 \\
\hline 53.1506119 & -27.7715912 & $20.51 \pm 0.04$ & $22.73 \pm 0.02$ & $22.24 \pm 0.02$ & $21.54 \pm 0.01$ & $21.26 \pm 0.01$ & $21.19 \pm 0.01$ & $22.62 \pm 0.07$ & $22.93 \pm 0.09$ & 0.23 & $\ldots$ & 4.67 \\
\hline 53.1512375 & -27.7986679 & $21.51 \pm 0.06$ & $25.86 \pm 0.09$ & $24.93 \pm 0.08$ & $24.37 \pm 0.04$ & $23.64 \pm 0.04$ & $23.83 \pm 0.06$ & $\ldots$ & $\ldots$ & 0.47 & $\ldots$ & 6.00 \\
\hline 53.1516838 & -27.7964039 & $22.93 \pm 0.11$ & $\ldots$ & $23.77 \pm 0.04$ & $23.49 \pm 0.03$ & $23.35 \pm 0.05$ & $23.15 \pm 0.05$ & $\ldots$ & $\cdots$ & 1.56 & $\ldots$ & 5.67 \\
\hline $53.1518784^{c}$ & -27.7754364 & $21.97 \pm 0.05$ & $\cdots$ & $23.76 \pm 0.05$ & $23.36 \pm 0.03$ & $22.58 \pm 0.03$ & $22.17 \pm 0.02$ & $22.93 \pm 0.06$ & $\cdots$ & 1.00 & $\cdots$ & 3.33 \\
\hline $53.1518898^{c}$ & -27.7828751 & $22.73 \pm 0.04$ & $\cdots$ & $24.44 \pm 0.04$ & $24.14 \pm 0.03$ & $23.45 \pm 0.03$ & $23.23 \pm 0.03$ & $24.11 \pm 0.13$ & $\cdots$ & 0.71 & $\cdots$ & 4.00 \\
\hline $53.1518974^{\mathrm{c}}$ & -27.7819862 & $23.17 \pm 0.08$ & $\ldots$ & $24.60 \pm 0.06$ & $24.32 \pm 0.04$ & $23.72 \pm 0.05$ & $23.32 \pm 0.04$ & $24.19 \pm 0.18$ & $\ldots$ & 0.84 & $\ldots$ & 4.00 \\
\hline $53.1520653^{c}$ & -27.7747822 & $21.65 \pm 0.03$ & $\cdots$ & $22.64 \pm 0.02$ & $22.15 \pm 0.01$ & $21.37 \pm 0.01$ & $21.16 \pm 0.01$ & $22.93 \pm 0.06$ & $\cdots$ & 0.70 & $\ldots$ & 3.67 \\
\hline $53.1523628^{\mathrm{c}}$ & -27.7779751 & $22.99 \pm 0.06$ & $\cdots$ & $24.71 \pm 0.08$ & $24.84 \pm 0.07$ & $24.96 \pm 0.16$ & $24.29 \pm 0.10$ & $22.93 \pm 0.06$ & $\ldots$ & 1.55 & $\ldots$ & 6.00 \\
\hline $53.1528244^{c}$ & -27.7826958 & $23.57 \pm 0.06$ & $28.01 \pm 0.26$ & $25.52 \pm 0.10$ & $25.21 \pm 0.07$ & $24.61 \pm 0.09$ & $24.25 \pm 0.08$ & $24.11 \pm 0.13$ & $\ldots$ & 0.70 & $\ldots$ & 6.00 \\
\hline 53.1531296 & -27.8120804 & $21.04 \pm 0.04$ & $\ldots$ & $23.85 \pm 0.04$ & $23.38 \pm 0.02$ & $23.30 \pm 0.04$ & $23.33 \pm 0.05$ & $24.11 \pm 0.12$ & $24.21 \pm 0.10$ & 0.07 & 0.2128 & 6.00 \\
\hline 53.1536751 & -27.8089409 & $22.06 \pm 0.06$ & $25.89 \pm 0.14$ & $24.92 \pm 0.08$ & $24.12 \pm 0.03$ & $23.80 \pm 0.05$ & $23.62 \pm 0.05$ & $\ldots$ & $\ldots$ & 0.29 & $\ldots$ & 5.33 \\
\hline 53.1546783 & -27.7932301 & $21.29 \pm 0.04$ & $23.86 \pm 0.03$ & $23.44 \pm 0.02$ & $22.86 \pm 0.01$ & $22.65 \pm 0.02$ & $22.62 \pm 0.02$ & $23.18 \pm 0.09$ & $23.79 \pm 0.09$ & 0.41 & $\ldots$ & 6.00 \\
\hline 53.1552696 & -27.7695465 & $21.67 \pm 0.06$ & $\ldots$ & $23.65 \pm 0.05$ & $23.07 \pm 0.02$ & $22.38 \pm 0.03$ & $22.13 \pm 0.03$ & $\ldots$ & $\ldots$ & 0.71 & 0.7359 & 3.67 \\
\hline 53.1556816 & -27.7793083 & $23.96 \pm 0.09$ & $\cdots$ & $23.99 \pm 0.05$ & $23.68 \pm 0.03$ & $23.28 \pm 0.04$ & $23.16 \pm 0.04$ & $\cdots$ & $\ldots$ & 1.58 & $\ldots$ & 4.00 \\
\hline $53.1559105^{\mathrm{c}}$ & -27.7948895 & $21.82 \pm 0.08$ & $\cdots$ & $24.02 \pm 0.04$ & $23.79 \pm 0.03$ & $23.43 \pm 0.05$ & $23.11 \pm 0.04$ & $24.63 \pm 0.12$ & $\cdots$ & 0.91 & $\ldots$ & 5.67 \\
\hline 53.1564293 & -27.8107758 & $20.45 \pm 0.02$ & $24.69 \pm 0.09$ & $22.20 \pm 0.02$ & $21.62 \pm 0.01$ & $20.97 \pm 0.01$ & $20.84 \pm 0.01$ & $22.90 \pm 0.08$ & $\cdots$ & 0.63 & 0.6650 & 3.67 \\
\hline $53.1567726^{\mathrm{c}}$ & -27.7955532 & $22.17 \pm 0.07$ & $\ldots$ & $23.79 \pm 0.04$ & $23.31 \pm 0.02$ & $22.66 \pm 0.03$ & $22.08 \pm 0.02$ & $23.34 \pm 0.15$ & $\cdots$ & 1.09 & 1.0970 & 3.33 \\
\hline 53.1572227 & -27.7785568 & $22.78 \pm 0.06$ & $\cdots$ & $24.59 \pm 0.05$ & $24.53 \pm 0.04$ & $24.41 \pm 0.07$ & $23.83 \pm 0.05$ & $\cdots$ & $\cdots$ & 0.76 & 1.3070 & 6.00 \\
\hline 53.1578369 & -27.7974815 & $22.27 \pm 0.10$ & $\cdots$ & $23.62 \pm 0.03$ & $23.26 \pm 0.02$ & $22.61 \pm 0.03$ & $22.40 \pm 0.03$ & $23.34 \pm 0.15$ & $\cdots$ & 0.70 & $\cdots$ & 4.00 \\
\hline 53.1580658 & -27.7692299 & $21.92 \pm 0.02$ & $\cdots$ & $21.18 \pm 0.01$ & $20.40 \pm 0.00$ & $20.02 \pm 0.01$ & $19.91 \pm 0.00$ & $23.79 \pm 0.14$ & $\cdots$ & 0.11 & $\cdots$ & 3.00 \\
\hline 53.1581879 & -27.7811279 & $21.00 \pm 0.02$ & $24.10 \pm 0.04$ & $22.93 \pm 0.02$ & $22.43 \pm 0.01$ & $21.93 \pm 0.02$ & $21.90 \pm 0.02$ & $23.22 \pm 0.09$ & $25.13 \pm 0.34$ & 0.44 & $\cdots$ & 5.67 \\
\hline
\end{tabular}


Table 1

(Continued)

\begin{tabular}{|c|c|c|c|c|c|c|c|c|c|c|c|c|}
\hline $\begin{array}{l}\text { R.A. } \\
(\mathrm{deg})\end{array}$ & $\begin{array}{l}\text { Decl. } \\
(\mathrm{deg})\end{array}$ & $U_{\text {mag }}$ & $\mathrm{FUV}_{\mathrm{mag}}$ & $B_{\text {mag }}$ & $V_{\text {mag }}$ & $i_{\mathrm{mag}}$ & $z_{\mathrm{mag}}$ & $\begin{array}{c}\text { GALEX } \\
\mathrm{NUV}_{\mathrm{mag}}\end{array}$ & $\begin{array}{l}\text { GALEX } \\
\text { FUV }_{\text {mag }}\end{array}$ & $z_{\text {phot }}$ & $z_{\text {spec }}$ & $\mathrm{ST}$ \\
\hline 53.1583176 & -27.7774792 & $22.76 \pm 0.04$ & $\ldots$ & $24.77 \pm 0.06$ & $24.79 \pm 0.05$ & $24.75 \pm 0.09$ & $24.64 \pm 0.10$ & $\ldots$ & $\ldots$ & 1.88 & $\ldots$ & 6.00 \\
\hline 53.1587791 & -27.7705669 & $22.47 \pm 0.04$ & $\ldots$ & $23.90 \pm 0.04$ & $23.56 \pm 0.03$ & $22.84 \pm 0.03$ & $22.45 \pm 0.02$ & $23.86 \pm 0.22$ & $\ldots$ & 0.91 & $\ldots$ & 3.67 \\
\hline 53.1599007 & -27.7668762 & $23.39 \pm 0.27$ & $\cdots$ & $24.76 \pm 0.07$ & $24.72 \pm 0.06$ & $24.02 \pm 0.07$ & $23.59 \pm 0.06$ & $21.86 \pm 0.04$ & $\cdots$ & 0.90 & $\cdots$ & 4.00 \\
\hline 53.1604424 & -27.7903595 & $23.03 \pm 0.07$ & $\ldots$ & $24.01 \pm 0.04$ & $24.01 \pm 0.03$ & $23.92 \pm 0.05$ & $23.92 \pm 0.06$ & $\ldots$ & $\ldots$ & 1.82 & $\ldots$ & 6.00 \\
\hline 53.1604996 & -27.7863064 & $23.83 \pm 0.07$ & $\ldots$ & $25.50 \pm 0.15$ & $25.36 \pm 0.11$ & $24.55 \pm 0.11$ & $24.28 \pm 0.10$ & $\ldots$ & $\ldots$ & 0.90 & $\ldots$ & 4.00 \\
\hline 53.1608238 & -27.7753963 & $21.70 \pm 0.06$ & $\ldots$ & $23.44 \pm 0.04$ & $22.50 \pm 0.02$ & $21.67 \pm 0.02$ & $21.42 \pm 0.02$ & $22.44 \pm 0.06$ & $23.02 \pm 0.10$ & 0.64 & $\ldots$ & 3.00 \\
\hline 53.1613579 & -27.7957401 & $22.93 \pm 0.10$ & $\ldots$ & $24.48 \pm 0.06$ & $24.06 \pm 0.04$ & $23.77 \pm 0.06$ & $23.54 \pm 0.06$ & $24.62 \pm 0.25$ & $25.37 \pm 0.24$ & 1.00 & $\ldots$ & 5.67 \\
\hline $53.1615295^{\mathrm{c}}$ & -27.7676716 & $22.95 \pm 0.10$ & $26.58 \pm 0.15$ & $24.96 \pm 0.07$ & $24.45 \pm 0.04$ & $23.78 \pm 0.05$ & $23.70 \pm 0.05$ & $21.86 \pm 0.00$ & $\ldots$ & 0.69 & $\ldots$ & 4.00 \\
\hline $53.1615944^{\mathrm{a}}$ & -27.7922535 & $20.89 \pm 0.02$ & $22.65 \pm 0.02$ & $21.81 \pm 0.01$ & $21.37 \pm 0.01$ & $21.02 \pm 0.01$ & $20.77 \pm 0.01$ & $21.98 \pm 0.04$ & $23.08 \pm 0.09$ & 0.42 & $\ldots$ & 5.33 \\
\hline $53.1619873^{c}$ & -27.7925415 & $21.63 \pm 0.04$ & $24.74 \pm 0.05$ & $24.91 \pm 0.04$ & $24.16 \pm 0.02$ & $23.75 \pm 0.03$ & $23.71 \pm 0.03$ & $21.97 \pm 0.03$ & $22.95 \pm 0.04$ & 0.25 & $\cdots$ & 5.00 \\
\hline $53.1619949^{c}$ & -27.7739410 & $21.87 \pm 0.06$ & $23.95 \pm 0.04$ & $23.58 \pm 0.03$ & $22.83 \pm 0.02$ & $22.62 \pm 0.02$ & $22.53 \pm 0.03$ & $22.39 \pm 0.04$ & $23.12 \pm 0.05$ & 0.26 & $\cdots$ & 5.67 \\
\hline $53.1623802^{\mathrm{c}}$ & -27.7750893 & $21.16 \pm 0.03$ & $23.66 \pm 0.03$ & $22.45 \pm 0.02$ & $21.48 \pm 0.01$ & $20.97 \pm 0.01$ & $20.69 \pm 0.01$ & $22.39 \pm 0.04$ & $23.12 \pm 0.05$ & 0.37 & $\cdots$ & 3.00 \\
\hline 53.1624947 & -27.7709045 & $24.45 \pm 0.15$ & $\cdots$ & $25.46 \pm 0.11$ & $25.10 \pm 0.07$ & $24.61 \pm 0.09$ & $24.45 \pm 0.09$ & $23.86 \pm 0.22$ & $\ldots$ & 1.13 & $\cdots$ & 6.00 \\
\hline $53.1628456^{\mathrm{a}, \mathrm{c}}$ & -27.7672405 & $21.16 \pm 0.03$ & $\ldots$ & $21.17 \pm 0.01$ & $20.94 \pm 0.01$ & $20.87 \pm 0.01$ & $20.84 \pm 0.01$ & $21.96 \pm 0.03$ & $\ldots$ & 0.05 & $\ldots$ & 6.00 \\
\hline 53.1635971 & -27.7935085 & $21.89 \pm 0.07$ & $\cdots$ & $25.17 \pm 0.07$ & $25.09 \pm 0.05$ & $24.98 \pm 0.08$ & $24.47 \pm 0.07$ & $\cdots$ & $\cdots$ & 1.54 & $\cdots$ & 6.00 \\
\hline 53.1641121 & -27.7873249 & $23.08 \pm 0.05$ & $\cdots$ & $24.95 \pm 0.07$ & $24.78 \pm 0.05$ & $24.32 \pm 0.07$ & $24.00 \pm 0.06$ & $\ldots$ & $\cdots$ & 0.75 & $\ldots$ & 6.00 \\
\hline 53.1648941 & -27.7787838 & $23.27 \pm 0.12$ & $\cdots$ & $25.31 \pm 0.10$ & $25.34 \pm 0.08$ & $24.89 \pm 0.11$ & $24.63 \pm 0.11$ & $\cdots$ & $\cdots$ & 1.26 & $\cdots$ & 6.00 \\
\hline 53.1659012 & -27.7815647 & $22.96 \pm 0.05$ & $\cdots$ & $24.67 \pm 0.08$ & $24.05 \pm 0.04$ & $23.29 \pm 0.04$ & $22.79 \pm 0.03$ & $\cdots$ & $\cdots$ & 0.95 & $\cdots$ & 3.33 \\
\hline 53.1661797 & -27.7875214 & $21.70 \pm 0.05$ & $\cdots$ & $23.02 \pm 0.03$ & $22.64 \pm 0.02$ & $21.98 \pm 0.02$ & $21.41 \pm 0.01$ & $\cdots$ & $\cdots$ & 1.04 & 1.0951 & 3.33 \\
\hline 53.1662140 & -27.7939320 & $21.99 \pm 0.10$ & $\cdots$ & $24.56 \pm 0.06$ & $24.54 \pm 0.04$ & $23.99 \pm 0.05$ & $23.63 \pm 0.04$ & $23.48 \pm 0.24$ & $\cdots$ & 0.71 & & 6.00 \\
\hline 53.1668854 & -27.7976780 & $22.72 \pm 0.06$ & $\cdots$ & $24.37 \pm 0.05$ & $24.43 \pm 0.04$ & $23.93 \pm 0.05$ & $23.75 \pm 0.05$ & $24.39 \pm 0.23$ & $\cdots$ & 1.18 & $\cdots$ & 6.00 \\
\hline $53.1675873^{\mathrm{c}}$ & -27.7925072 & $23.27 \pm 0.54$ & $\cdots$ & $23.76 \pm 0.04$ & $23.45 \pm 0.02$ & $23.01 \pm 0.03$ & $22.72 \pm 0.03$ & $23.48 \pm 0.24$ & $\cdots$ & 0.71 & $\cdots$ & 5.67 \\
\hline 53.1679382 & -27.7781277 & $23.47 \pm 0.12$ & $26.16 \pm 0.10$ & $24.86 \pm 0.08$ & $24.05 \pm 0.03$ & $23.70 \pm 0.05$ & $23.63 \pm 0.06$ & $\ldots$ & $\ldots$ & 0.26 & 0.7122 & 5.33 \\
\hline 53.1680222 & -27.7896690 & $22.09 \pm 0.07$ & $25.64 \pm 0.09$ & $24.12 \pm 0.04$ & $23.57 \pm 0.02$ & $22.98 \pm 0.03$ & $22.90 \pm 0.03$ & $24.86 \pm 0.14$ & & 0.50 & $\ldots$ & 4.33 \\
\hline $53.1680603^{c}$ & -27.8074017 & $22.91 \pm 0.11$ & $25.32 \pm 0.08$ & $24.77 \pm 0.09$ & $23.97 \pm 0.04$ & $23.72 \pm 0.06$ & $23.70 \pm 0.07$ & $24.35 \pm 0.17$ & $25.89 \pm 0.27$ & 0.41 & $\cdots$ & 6.00 \\
\hline 53.1681747 & -27.8128967 & $23.16 \pm 0.07$ & $\ldots$ & $24.43 \pm 0.06$ & $24.06 \pm 0.03$ & $23.35 \pm 0.04$ & $23.04 \pm 0.03$ & $\cdots$ & $\ldots$ & 0.83 & $\cdots$ & 3.67 \\
\hline 53.1699409 & -27.7710609 & $20.59 \pm 0.02$ & $\cdots$ & $22.01 \pm 0.02$ & $21.18 \pm 0.01$ & $20.37 \pm 0.01$ & $20.12 \pm 0.01$ & $22.94 \pm 0.05$ & $25.26 \pm 0.20$ & 0.65 & $\cdots$ & 3.00 \\
\hline 53.1703148 & -27.7852764 & $23.07 \pm 0.09$ & $25.32 \pm 0.07$ & $24.86 \pm 0.07$ & $24.15 \pm 0.03$ & $23.90 \pm 0.04$ & $23.67 \pm 0.04$ & $\cdots$ & $\cdots$ & 0.29 & 0.7122 & 5.67 \\
\hline $53.1704826^{\mathrm{b}}$ & -27.7613792 & $21.58 \pm 0.03$ & $\cdots$ & $22.53 \pm 0.01$ & $21.84 \pm 0.01$ & $21.49 \pm 0.01$ & $21.39 \pm 0.01$ & $23.16 \pm 0.09$ & $23.91 \pm 0.09$ & 0.25 & $\cdots$ & 4.67 \\
\hline 53.1705437 & -27.8065834 & $22.75 \pm 0.09$ & $\cdots$ & $24.65 \pm 0.06$ & $24.67 \pm 0.05$ & $24.42 \pm 0.07$ & $23.96 \pm 0.06$ & $24.74 \pm 0.47$ & $\cdots$ & 0.77 & 1.2441 & 6.00 \\
\hline $53.1706848^{\mathrm{b}, \mathrm{c}}$ & -27.7579365 & $22.42 \pm 0.05$ & $\cdots$ & $24.19 \pm 0.08$ & $23.56 \pm 0.03$ & $22.98 \pm 0.03$ & $22.93 \pm 0.04$ & $22.77 \pm 0.06$ & $23.16 \pm 0.10$ & 0.27 & $\cdots$ & 5.00 \\
\hline 53.1707726 & -27.8046780 & $23.14 \pm 0.09$ & $\cdots$ & $24.06 \pm 0.05$ & $23.91 \pm 0.04$ & $23.71 \pm 0.06$ & $23.63 \pm 0.07$ & $25.04 \pm 0.24$ & $\cdots$ & 1.79 & $\cdots$ & 6.00 \\
\hline $53.1713791^{\mathrm{b}, \mathrm{c}}$ & -27.7574749 & $20.96 \pm 0.02$ & $\cdots$ & $21.69 \pm 0.02$ & $21.07 \pm 0.01$ & $20.71 \pm 0.01$ & $20.63 \pm 0.01$ & $22.70 \pm 0.04$ & $23.23 \pm 0.05$ & 0.25 & $\cdots$ & 5.33 \\
\hline $53.1721077^{\mathrm{c}}$ & -27.7969379 & $23.11 \pm 0.13$ & $25.21 \pm 0.06$ & $24.58 \pm 0.08$ & $23.71 \pm 0.03$ & $23.42 \pm 0.05$ & $23.32 \pm 0.05$ & $22.94 \pm 0.05$ & $23.50 \pm 0.06$ & 0.19 & $\cdots$ & 3.33 \\
\hline
\end{tabular}


Table 1

(Continued)

\begin{tabular}{|c|c|c|c|c|c|c|c|c|c|c|c|c|}
\hline $\begin{array}{l}\text { R.A. } \\
(\operatorname{deg})\end{array}$ & $\begin{array}{l}\text { Decl. } \\
(\mathrm{deg})\end{array}$ & $U_{\text {mag }}$ & $\mathrm{FUV}_{\mathrm{mag}}$ & $B_{\text {mag }}$ & $V_{\text {mag }}$ & $i_{\mathrm{mag}}$ & $z_{\mathrm{mag}}$ & $\begin{array}{c}\text { GALEX } \\
\mathrm{NUV}_{\mathrm{mag}} \\
\end{array}$ & $\begin{array}{l}\text { GALEX } \\
\text { FUV }_{\mathrm{mag}}\end{array}$ & $z_{\text {phot }}$ & $z_{\text {spec }}$ & ST \\
\hline 53.1721840 & -27.8058681 & $22.21 \pm 0.06$ & $\cdots$ & $23.94 \pm 0.06$ & $23.83 \pm 0.04$ & $23.71 \pm 0.08$ & $23.24 \pm 0.06$ & $\cdots$ & $\cdots$ & 0.77 & 1.3180 & 6.00 \\
\hline 53.1722603 & -27.7651482 & $22.41 \pm 0.08$ & $\cdots$ & $23.34 \pm 0.04$ & $22.64 \pm 0.02$ & $22.01 \pm 0.02$ & $21.88 \pm 0.02$ & $23.98 \pm 0.16$ & $24.65 \pm 0.25$ & 0.51 & $\cdots$ & 3.67 \\
\hline $53.1725159^{c}$ & -27.7963371 & $21.05 \pm 0.06$ & $23.66 \pm 0.03$ & $23.01 \pm 0.03$ & $22.15 \pm 0.01$ & $21.85 \pm 0.02$ & $21.67 \pm 0.02$ & $22.89 \pm 0.08$ & $23.27 \pm 0.10$ & 0.29 & 0.3469 & 3.67 \\
\hline 53.1726112 & -27.7809887 & $23.37 \pm 0.15$ & & $24.04 \pm 0.06$ & $22.90 \pm 0.02$ & $22.05 \pm 0.02$ & $21.71 \pm 0.01$ & $\cdots$ & $\cdots$ & 0.66 & $\cdots$ & 2.67 \\
\hline 53.1730003 & -27.7779026 & $22.08 \pm 0.06$ & $\cdots$ & $24.06 \pm 0.04$ & $23.98 \pm 0.03$ & $23.53 \pm 0.04$ & $23.20 \pm 0.04$ & $24.46 \pm 0.26$ & $\ldots$ & 0.71 & $\ldots$ & 6.00 \\
\hline $53.1730042^{\mathrm{b}, \mathrm{c}}$ & -27.7590351 & $20.62 \pm 0.02$ & $\ldots$ & $21.51 \pm 0.01$ & $20.76 \pm 0.01$ & $20.37 \pm 0.01$ & $20.26 \pm 0.01$ & $22.69 \pm 0.06$ & $22.99 \pm 0.08$ & 0.26 & $\ldots$ & 5.00 \\
\hline 53.1747513 & -27.7992420 & $19.58 \pm 0.03$ & $22.00 \pm 0.02$ & $21.14 \pm 0.01$ & $20.54 \pm 0.01$ & $20.25 \pm 0.01$ & $20.14 \pm 0.01$ & $22.04 \pm 0.03$ & $22.30 \pm 0.03$ & 0.26 & 0.1514 & 5.67 \\
\hline 53.1761894 & -27.7961178 & $21.25 \pm 0.06$ & & $22.87 \pm 0.02$ & $22.36 \pm 0.01$ & $21.62 \pm 0.01$ & $21.23 \pm 0.01$ & $24.28 \pm 0.18$ & $\ldots$ & 0.86 & 0.9961 & 3.67 \\
\hline $53.1767311^{\mathrm{d}}$ & -27.7996502 & $22.89 \pm 0.12$ & & $22.53 \pm 0.02$ & $20.58 \pm 0.00$ & $18.93 \pm 0.00$ & $18.32 \pm 0.00$ & $\cdots$ & $\ldots$ & 0.70 & $\ldots$ & 1.33 \\
\hline 53.1777191 & -27.7869625 & $22.52 \pm 0.07$ & & $25.15 \pm 0.08$ & $25.08 \pm 0.07$ & $24.52 \pm 0.08$ & $24.41 \pm 0.08$ & $\cdots$ & $\ldots$ & 1.12 & $\ldots$ & 6.00 \\
\hline $53.1778793^{b}$ & -27.7577496 & $23.50 \pm 0.12$ & $\cdots$ & $25.02 \pm 0.05$ & $25.05 \pm 0.05$ & $24.44 \pm 0.06$ & $24.27 \pm 0.06$ & $\cdots$ & $\cdots$ & 0.91 & $\cdots$ & 6.00 \\
\hline 53.1782227 & -27.7830944 & $21.45 \pm 0.05$ & $\cdots$ & $24.03 \pm 0.05$ & $23.84 \pm 0.03$ & $23.45 \pm 0.04$ & $22.92 \pm 0.03$ & $\cdots$ & $\cdots$ & 0.77 & $\cdots$ & 5.67 \\
\hline 53.1784172 & -27.7682304 & $21.09 \pm 0.05$ & $\cdots$ & $22.39 \pm 0.02$ & $21.54 \pm 0.01$ & $20.68 \pm 0.01$ & $20.39 \pm 0.01$ & $23.12 \pm 0.07$ & $\ldots$ & 0.72 & $\ldots$ & 3.00 \\
\hline $53.1799660^{\mathrm{b}}$ & -27.7573910 & $23.55 \pm 0.13$ & $\ldots$ & $25.28 \pm 0.08$ & $24.76 \pm 0.05$ & $24.31 \pm 0.06$ & $24.33 \pm 0.08$ & $\ldots$ & $\ldots$ & 0.68 & $\ldots$ & 6.00 \\
\hline 53.1815453 & -27.7879925 & $22.07 \pm 0.04$ & $23.86 \pm 0.03$ & $23.94 \pm 0.04$ & $23.48 \pm 0.02$ & $23.35 \pm 0.03$ & $23.44 \pm 0.04$ & $23.57 \pm 0.14$ & $23.55 \pm 0.19$ & 0.07 & 0.2122 & 6.00 \\
\hline 53.1821976 & -27.7939968 & $23.18 \pm 0.09$ & $27.64 \pm 0.23$ & $25.70 \pm 0.09$ & $25.59 \pm 0.06$ & $25.07 \pm 0.08$ & $24.93 \pm 0.08$ & $\cdots$ & $\ldots$ & 1.13 & $\cdots$ & 6.00 \\
\hline $53.1826210^{\mathrm{d}}$ & -27.7681408 & $19.44 \pm 0.00$ & $\cdots$ & $\cdots$ & $\cdots$ & $\cdots$ & $17.45 \pm 0.00$ & $\cdots$ & $\cdots$ & 0.30 & $\cdots$ & 6.00 \\
\hline $53.1841164^{\mathrm{b}}$ & -27.7559299 & $22.82 \pm 0.13$ & $\ldots$ & $24.89 \pm 0.06$ & $24.73 \pm 0.05$ & $24.41 \pm 0.07$ & $24.33 \pm 0.08$ & $\ldots$ & $\ldots$ & 1.89 & $\ldots$ & 6.00 \\
\hline 53.1841660 & -27.7926407 & $21.36 \pm 0.02$ & $25.92 \pm 0.13$ & $22.90 \pm 0.02$ & $22.33 \pm 0.01$ & $21.65 \pm 0.01$ & $21.44 \pm 0.01$ & $\ldots$ & $\ldots$ & 0.69 & 0.7372 & 3.67 \\
\hline 53.1841698 & -27.7915535 & $23.38 \pm 0.10$ & $\cdots$ & $24.95 \pm 0.14$ & $24.48 \pm 0.07$ & $23.77 \pm 0.07$ & $23.36 \pm 0.06$ & $\ldots$ & $\ldots$ & 1.45 & $\ldots$ & 3.00 \\
\hline $53.1855202^{\mathrm{b}}$ & -27.7554283 & $22.01 \pm 0.07$ & $\ldots$ & $23.96 \pm 0.03$ & $23.07 \pm 0.01$ & $22.52 \pm 0.02$ & $22.36 \pm 0.02$ & $\ldots$ & $\ldots$ & 0.43 & 0.5328 & 3.33 \\
\hline $53.1869583^{c}$ & -27.7910004 & $19.03 \pm 0.01$ & $21.67 \pm 0.01$ & $20.21 \pm 0.01$ & $19.19 \pm 0.00$ & $18.65 \pm 0.00$ & $18.44 \pm 0.00$ & $21.32 \pm 0.02$ & $21.89 \pm 0.02$ & 0.23 & $\cdots$ & 2.33 \\
\hline $53.1877899^{c}$ & -27.7940979 & $19.83 \pm 0.02$ & $22.23 \pm 0.02$ & $21.49 \pm 0.01$ & $20.68 \pm 0.01$ & $20.45 \pm 0.01$ & $20.23 \pm 0.01$ & $21.95 \pm 0.02$ & $22.49 \pm 0.03$ & 0.27 & 0.3446 & 3.67 \\
\hline $53.1879463^{b}$ & -27.7615261 & $21.55 \pm 0.06$ & $\ldots$ & $21.50 \pm 0.01$ & $20.61 \pm 0.01$ & $20.22 \pm 0.01$ & $20.05 \pm 0.01$ & $23.59 \pm 0.11$ & $24.47 \pm 0.21$ & 0.22 & $\ldots$ & 3.33 \\
\hline $53.1879768^{\mathrm{a}, \mathrm{c}}$ & -27.7900066 & $21.10 \pm 0.03$ & $24.86 \pm 0.06$ & $22.69 \pm 0.02$ & $21.53 \pm 0.01$ & $20.79 \pm 0.01$ & $20.39 \pm 0.01$ & $21.32 \pm 0.02$ & $21.89 \pm 0.02$ & 0.51 & 0.4357 & 2.33 \\
\hline $53.1898384^{\mathrm{b}}$ & -27.7588539 & $20.64 \pm 0.03$ & & $21.84 \pm 0.01$ & $21.41 \pm 0.01$ & $21.17 \pm 0.01$ & $21.17 \pm 0.01$ & $22.43 \pm 0.04$ & $22.65 \pm 0.03$ & 0.24 & $\cdots$ & 6.00 \\
\hline $53.1901550^{\mathrm{b}}$ & -27.7651958 & $20.49 \pm 0.06$ & & $22.01 \pm 0.02$ & $20.95 \pm 0.01$ & $20.52 \pm 0.01$ & $20.29 \pm 0.01$ & $22.82 \pm 0.07$ & $23.69 \pm 0.13$ & 0.36 & $\cdots$ & 3.00 \\
\hline
\end{tabular}

\section{Notes.}

${ }^{a}$ X-ray source Koekemoer et al. (2004).

${ }^{\mathrm{b}}$ Source is outside the UDF footprint.

${ }^{c}$ Confusion in GALEX image.

${ }^{\mathrm{d}}$ Star. 


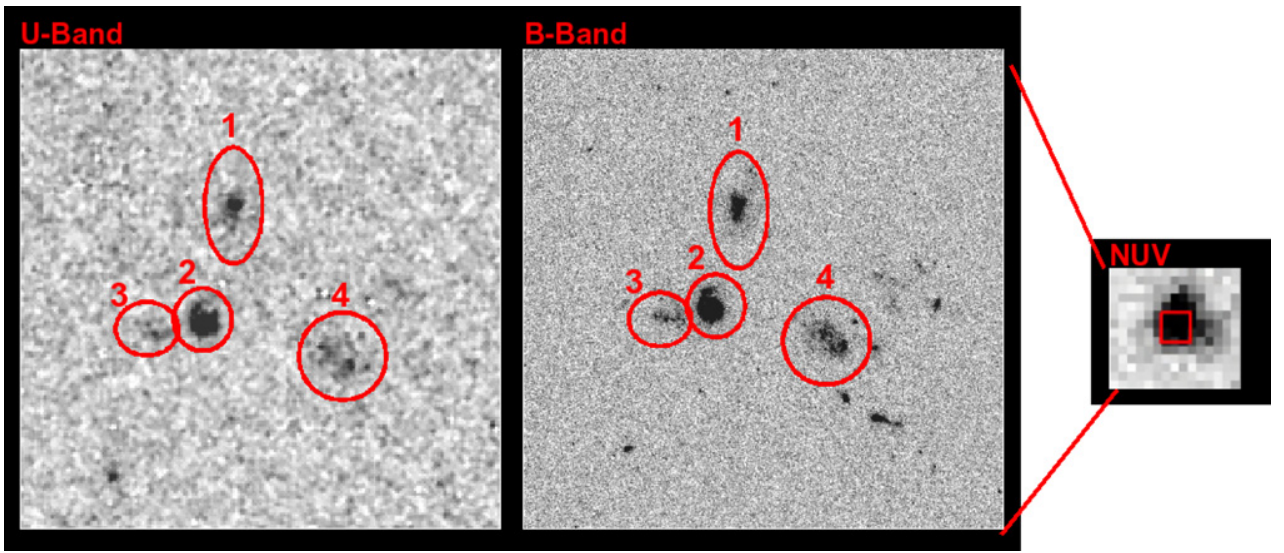

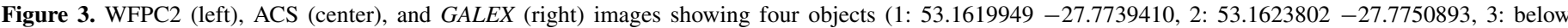
magnitude limit of the $U$-band catalog, 4: 53.1608238 -27.7753963) which are within the GALEX beam. All three images are $20^{\prime \prime} \times 20^{\prime \prime}$.

(A color version of this figure is available in the online journal.)

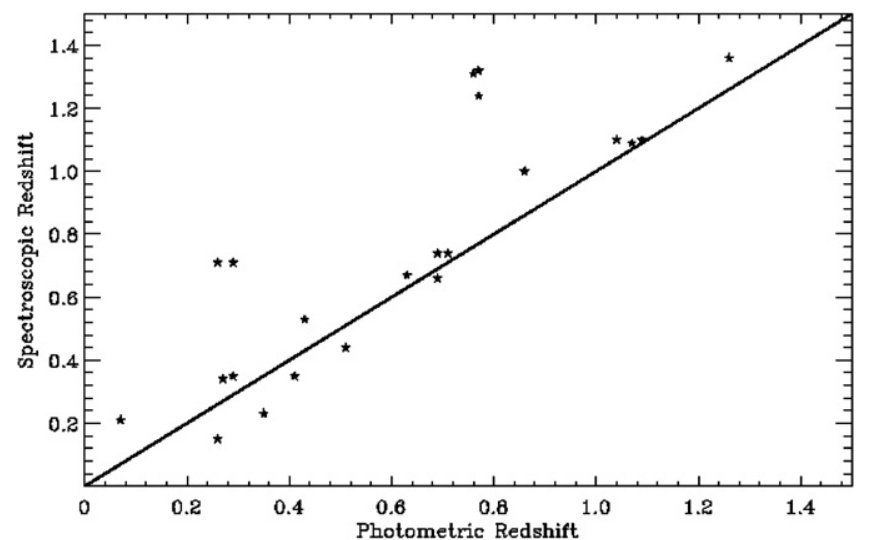

Figure 4. Photometric redshifts from GOODS vs. spectroscopic redshifts from the ESO/GOODS Chandra Deep Field-South spectroscopy masters catalog.

resolution is significantly lower than HST's (GOODS ACS image has $0^{\prime \prime} .03$ pixel $^{-1}$ ), we have searched for objects where confusion might be problematic and one should use the GALEX data with caution. We found 25 objects where confusion was an important factor in the NUV and FUV and have flagged them in the catalog. In Figure 3, we show an example of a single GALEX NUV detection of at least four identified objects in the $U$ and $B$ band.

\section{THE CATALOG}

Table 1 presents the $U$-band catalog of the UDF NUV sources. Columns 1 and 2 are the GOODS World Coordinate System (WCS) right ascension and declination in degrees. Three objects with Chandra X-ray detections (Koekemoer et al. 2004) are flagged as "a". ${ }^{8}$ Columns 3-8 are the $U$, FUV, $B, V$, $i$, and $z$ magnitudes and photometric errors ( $\mathrm{MAG}_{-}$AUTO), respectively. The $B, V, i$, and $z$ magnitudes were obtained from the GOODS$\mathrm{S}$ B-selected catalog. Note that not all $U$-band detections have FUV photometry because they are either outside the ACS/SBC footprint, are nondetections $(\mathrm{S} / \mathrm{N}<3 \sigma)$, or are not resolved in the FUV imaging. Columns 9 and 10 are GALEX NUV and FUV magnitudes from the GR4. Sources with confusion are flagged as "c" in Column 1. Columns 11 and 12 list photometric redshifts $\left(z_{\text {phot }}\right)$ for all objects, and available spectroscopic redshifts

\footnotetext{
8 Seven other Chandra X-ray sources found in the UDF were not detected in
} our $U$-band image. $\left(z_{\text {spec }}\right)$ for 21 objects. The $z_{\text {spec }}$ are from the GOODS collaboration (taken from the ESO/GOODS-S spectroscopy masters catalog ${ }^{9}$ ), and Figure 4 plots $z_{\text {spec }}$ as a function of $z_{\text {phot }}$ for these objects. Column 14 are spectral types from the GOODS Bselected catalog based on spectral energy distributions (SED) from Coleman et al. (1980) and Kinney et al. (1996). Type 1 galaxies are early-types (E, S0, and Sa), type 2 are Sbc, type 3 are Scd, type 4 are irregulars, and types 5 and 6 are starbursts SB1 and SB2. The SED templates of SB1's and SB2's are differentiated by the values of intrinsic color excess, $E(B-V)$. SB1 has $E(B-V) \leqslant 0.10$ and SB2 has $0.11 \leqslant E(B-V) \leqslant 0.21$ (Kinney et al. 1996). The $U$-band catalog, including $U$, FUV, $B$, and $B V i$ postage stamp images of each source, is available online at http://goods.gsfc.nasa.gov/release/UDF_F300W/original/ gallery/udf_u_fuv.html.

We are grateful to the GOODS team. Support for this work was provided by NASA through grants HST-GO-10403.13A and HST-GO-10632.03A from the Space Telescope Science Institute, which is operated by the Association of Universities for Research in Astronomy, Inc., under NASA contract NAS526555. D.F.d.M. and E.V. were funded by NASA research grants NNG05GG06G and NNX08AR95H. We are grateful to Sara Petty for helping with the early stages of this project.

GALEX is a NASA's Small Explorer, launched in 2003 April. We gratefully acknowledge NASA's support for construction, operation, and science analysis for the GALEX mission, developed in cooperation with the Centre National d'Etudes Spatiales of France and the Korean Ministry of Science and Technology.

\section{REFERENCES}

Beckwith, S. V. W., et al. 2006, AJ, 132, 1729

Bertin, E., \& Arnouts, S. 1996, A\&AS, 117, 393

Casertano, S., et al. 2000, AJ, 120, 2747

Coleman, G. D., Wu, C.-C., \& Wedman, D. W. 1980, ApJS, 43, 393

Cowie, L. L., Songalia, A., Hu, E. M., \& Cohen, J. G. 1996, AJ, 112, 839

Dahlen, T., Mobasher, B., Dickinson, M., Ferguson, H. C., Giavalisoc, M., Kretchmer, C., \& Ravindranath, S. 2007, ApJ, 654, 172

de Mello, D. F., Dahlen, T., Gardner, J. P., \& Grogin, N. A. 2006a, AJ, 132, 2014

de Mello, D. F., Wadadekar, Y., Dahlen, T., Casertano, S., \& Gardner, J. P. 2006b, AJ, 131, 216

Giavalisco, M., et al. 2004, ApJ, 600, L93

Hopkins, A. M., \& Beacom, J. F. 2006, ApJ, 651, 142

\footnotetext{
9 See http://www.eso.org/science/goods/spectroscopy/CDFS_Mastercat.
} 
Kinney, A., Calzetti, D., Bohlin, R. C., McQuade, K., Storchi-Bergmann, T., \& Schmitt, H. 1996, ApJ, 467, 38

Koekemoer, A. M., Fruchter, A. S., Hook, R. N., \& Hack, W. 2002, in Proc. 2002 HST Calibration Workshop: Hubble after the Installation of the ACS and the NICMOS Cooling System, ed. S. Arribas, A. Koekemoer, \&

B. Whitmore (Baltimore, MD: Space Telescope Science Institute), 337

Koekemoer, A. M., et al. 2004, ApJ, 600, L123

Kron, R. G. 1980, ApJS, 43, 305
Morrissey, P., et al. 2007, ApJS, 173, 682

Mouri, H., \& Taniguchi, Y. 2006, A\&A, 459, 371

Neistein, E., van den Bosch, F. C., \& Dekel, A. 2006, MNRAS, 372, 933

Savaglio, S., et al. 2005, ApJ, 635, 260 S

Siana, B., et al. 2007, ApJ, 668, 62S

Teplitz, H. I., et al. 2006, AJ, 132, 853

Wadadekar, Y., Casertano, S., \& de Mello, D. F. 2006, AJ, 132, 1023

Williams, R., et al. 1996, AJ, 112, 1335 